\title{
Intelligence System for Error Analysis Based on the Takagi-Sugeno Model
}

\author{
NuHua Cheng ${ }^{1, a}$, Wei Lv²,b ${ }^{2}$ YuHua $\mathrm{Ni}^{3, \mathrm{c}}$, and Cai Yong ${ }^{4, \mathrm{~d}}$ \\ ${ }^{1}$ NuHua Cheng,Beijing Normal University, Zhuhai,519000, China \\ ${ }^{2}$ Wei Lv, Beijing Normal University, Zhuhai,519000, China \\ ${ }^{3}$ YuHua Ni, Beijing Normal University, Zhuhai,519000, China \\ ${ }^{4}$ Cai Yong, Beijing Normal University, Zhuhai,519000, China

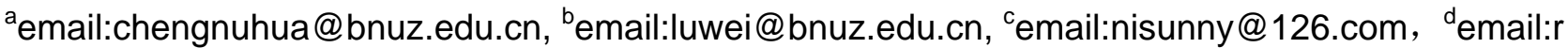 \\ ubeman@126.com
}

Keywords: Intelligence-system;Takagi-Sugeno;Cloud Computing;education ;data mining.

Abstract. Through the error from feedback of classroom teaching system ,based on the analysis and judgment of the intelligence system, the system can provide the cause of the error for students, and automatically push the error corresponding after-school classroom exercises , enhance learning relevance and learning efficiency.

\section{Introduction}

How to improve the direction of teachers teaching and students' enthusiasm and initiative for learning, to make teaching more with less, which is the main problem solved by us.

"Electronic classroom" and "Physical Studio" in American provide a certain reference value for our teaching . Man-machine dialogue is set in the teaching courseware, demanding students to think and answer all the questions in order to fully mobilize the enthusiasm of the students.

Some districts in Shanghai is currently organizing "electronic book- bag" and cloud computing to support the teaching experiment.

\section{Content of Study}

Architecture as shown in Fig. 1.

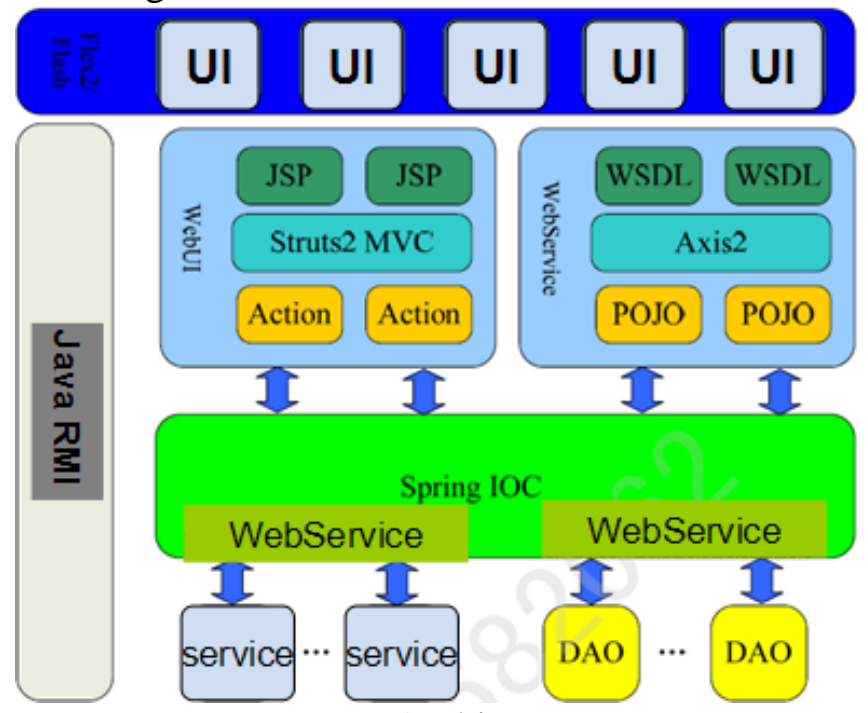

Fig. 1 Architecture

The structural model of learning elements, based on the learning courses for demand. 
Teachers, according to the results of interactive teaching, effect of student learning, based on public standard courseware, create the follow-up teaching materials, to support personalized preparation and teaching for teachers.

According to the results of interactions in classroom, students create a personal wrong titles and learning workbooks. In connection with the weak link for the students, correct only errors, practice only wrong titles, learning on demand, to break the tradition of the sea tactical and lighten the burden of student work. The same time, through the learning points and virtual rewards of cloud service platform, encourage students' enthusiasm for learning, and guide students to develop a good way to learn and good learning habits.

Intelligence-system based on Takagi-Sugeno

Intelligence-system is shown in Fig. 2.

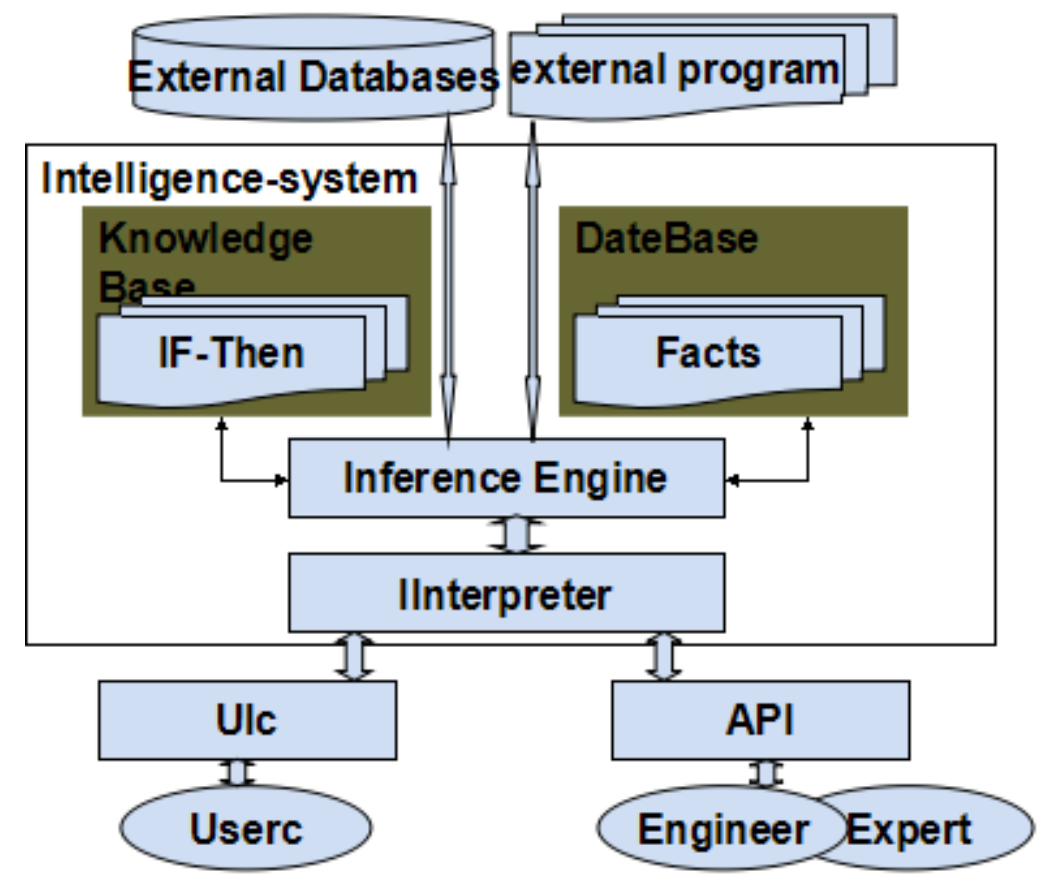

Fig. 2 Intelligence-system

T-S system, composed of $\mathrm{N}$ follows rules

Ri: If $s_{1}$ is $L_{1}{ }^{i}$ and $s_{2}$ is $L_{2}{ }^{i}$ and ... And $s_{n}$ is $L^{i}$

Then $\mathrm{y}^{\mathrm{i}}=$ pi $0+$ pi1s $1+\ldots+$ pinsn

$\mathrm{s}_{1}, \ldots, \mathrm{s}_{\mathrm{n}}$ as the input variables, $\mathrm{y}_{\mathrm{i}}$ as the I rule consequents output variables, $\mathrm{Li}^{1}, \ldots, \mathrm{Li}^{\mathrm{n}}$ as the triangle membership function,pi $0, \ldots$, pin as linear gain.

Output for T-S system, As the activation rule consequent weighted sum.

$y=\sum_{R i \in A}\left[\sum_{j=0}^{n} p_{i j} S_{j}\right]$

$\mathrm{TR}^{\mathrm{i}}$ as the rules of the true value, $\mathrm{A}$ as collection of all activated rules, $\mathrm{s} 0 \equiv 1$. 


\section{Platform}

Education cloud services platform is a cloud-based, distributed system architecture, RUP methodology to be used, case-driven, architecture-centric. The topological structure of the system is shown in Fig. 3.Based on service-oriented architecture (SOA) application development and integration, the ubiquitous education platform can be adapted to the students' new learning methods, learning content, and can also be adapted to parents, teachers, new requirements.

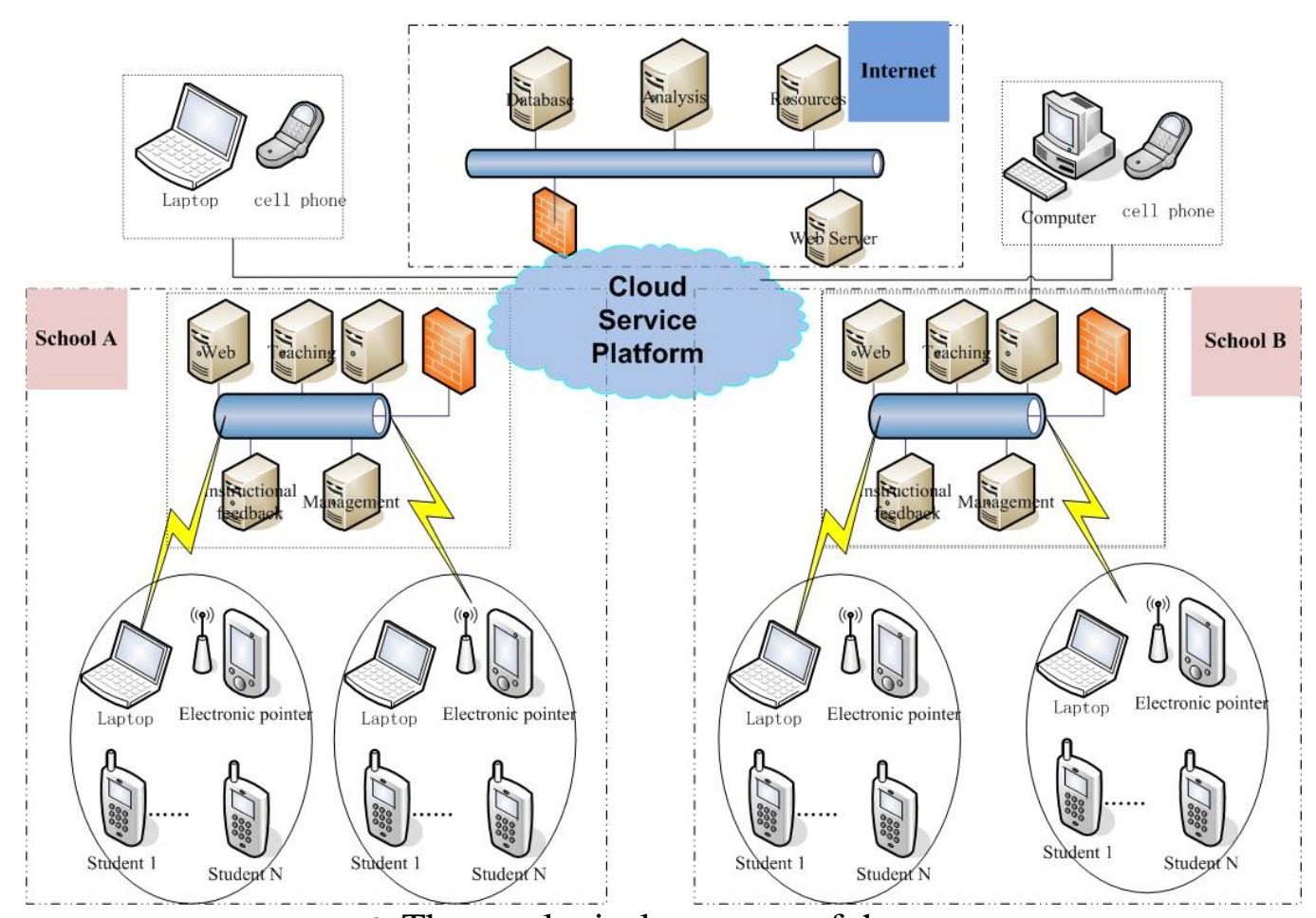

Fig. 3 The topological structure of the system

\section{Application}

In September 2011, the cloud service platform, the six experimental classes of the first primary in Xiangzhou , in Zhuhai City, Guangdong Province, are firstly selected to make experiments. In February 2012, extended to 24 classes of the lower grades. September 2012 ,to promote to 48 classes of the first primary in Xiangzhou . There are 2442 students and 127 teachers in the school. The teachers' classroom practice courseware 1930 have been uploaded, 52897 people download, over 57,831 platform patronage.

Platform pilot, 30\% reduction in the workload of teachers, and learning-targeted to be increased by $50 \%$, a $50 \%$ increase in enthusiasm for learning. There is a fewer and fewer students with learning difficulties. Feedback cycle of parents is two weeks or so in the past, now they can understand the learning effect every day. An assessment quality of teaching is made from a few months to the day. Students, parents, teachers, schools, feedback is good.

\section{Summary}

The system realizes the function of teaching and learning on demand, and is praised by platform users.

\section{Acknowledgements}

The paper is support by National Natural Science Foundation of China:11126039. 


\section{References}

[1] F. Chang, J. Dean, S. Ghemawat, W. C. Hsieh, D. A. Wallach, M. Burrows, T. Chandra, A. Fikes, and R. E. Gruber. Bigtable: A distributed storage system for structured data. In OSDI, 2006.

[2] R. L. Grossman and Y. Gu. Data mining using high performance clouds: Experimental studies using sector and sphere. In KDD, 2008.

[3] 2 Jouf f e L. Fu zzy inf erence sys t em l earning by reinf orcement meth od s. IE E E Tr ans . SMC —— Part C, Appl ications and Reviews , 1998, 28( 3): 338 355

[4] 3 Kim M S, Hong S G, Lee J J.On-line fu zzy Q-learning w i th ext ended rule and int erpolati on t ech nique. In: Proc.1999 IEEE /RS J Int . Conf. Int el ligent Robotics and Syst ems, Kyongju: South Korea, 1999, 2: 757 762

[5] 5 Sut t on R S, Bart o A G. Reinfo rcem ent Learning: An Int rod uct ion. M A: MI T Pres s, 1998

[6] 6 Yan X W, Deng Z D, Sun Z Q. Fuzzy advantage learning. In: Proc. IEEE, 9th Int. Conf. Fuzzy Syst em s, TX: SanAnt oni a, 2000, 2: 865 870 